\title{
Examination of weight status, parity and maternal education factors on intentions to breastfeed and breastfeeding duration in an Irish cohort.
}

\author{
Shauna Callaghan, Rebecca L. Moore, Aisling A. Geraghty, Cara Yelverton and \\ Prof. Fionnuala McAuliffe \\ UCD Perinatal Research Centre, School of Medicine, University College Dublin, National Maternity Hospital, Dublin, \\ Ireland
}

\section{Abstract}

Introduction

Breastfeeding can improve health outcomes for mothers and babies and is an important factor in the reduction of obesity. However, breastfeeding rates in Ireland are amongst the lowest internationally, with rates of $59.9 \%$ in 2016.

\section{Materials \& Methods}

The objective of this study was to assess intention to breastfeed at booking, feeding status at hospital discharge, one and three months postnatal and influencing factors.

Secondary data from 124 healthy pregnant women participating in an RCT in the National Maternity Hospital who recorded their intention to breastfeed at booking were included. Observational data including demographic and lifestyle data was collected from participants.

\section{Results}

Of the 124 women, $80.7 \%$ were Irish, $86.2 \%$ had achieved third level education, and $43.5 \%(\mathrm{n}=54)$ were multiparous. Mean age was 32.7 years and mean BMI $24.9 \mathrm{~kg} / \mathrm{m} 2.91 .1 \%(\mathrm{n}=113)$ reported intending to breastfeed at booking. Upon hospital discharge, $91 \%$ of those intending to breastfeed had initiated breastfeeding $(n=101 / 111) .78 .6 \%(n=77 / 98)$ and $66.7 \%(n=58 / 87)$ of those who had intended to breastfeed remained breastfeeding at one and three months postnatal respectively. Intention to breastfeed in early pregnancy was significantly higher amongst women with third level education $(p<0.001)$. Intention to breastfeed did not differ significantly with maternal age, ethnicity, BMI, or parity, however, women who had breastfed previously were more likely to intend to breastfeed for future infants $(\mathrm{p}<0.001)$. Women with higher education were more likely to breastfeed at discharge, one and three months postnatal $(\mathrm{p}<0.05)$. Although maternal BMI was not associated with intention to breastfeed, women who did not initiate breastfeeding at hospital discharge had a significantly higher BMI $(\mathrm{M}=27.22 \mathrm{~kg} / \mathrm{m} 2, \mathrm{SD}=4.53, \mathrm{p}=0.018)$ compared to women who initiated breastfeeding $(M=24.68 \mathrm{~kg} / \mathrm{m} 2, \mathrm{SD}=3.05)$. Similarly, of those intending to breastfeed, women who were not breastfeeding at three months postnatal had a significantly higher BMI $(\mathrm{M}=26.33 \mathrm{~kg} / \mathrm{m} 2, \mathrm{SD}=3.99, \mathrm{p}=0.01)$ than those continuing breastfeeding $(M=24.08 \mathrm{~kg} / \mathrm{m} 2, \mathrm{SD}=2.61)$. There were no significant differences regarding feeding status with maternal ethnicity, age, or parity at discharge and one month postnatal $(\mathrm{p}>0.05)$. However, primiparous women intending to breastfeed were significantly more likely to continue breastfeeding at three months compared to multiparous women $(\mathrm{p}<0.05)$.

\section{Discussion}

Many factors are shown to influence breastfeeding intention and duration including parity, education and BMI. It is important to identify these potentially inhibiting factors during pregnancy to develop support measures to encourage initiation and continuation of breastfeeding.

\section{Conflict of Interest}

There is no conflict of interest. 